\title{
Fiber-Optic Temperature Sensor Based on Temperature-Dependent Refractive Index of Germanium-Silica Coating Stack
}

\author{
Chunxian Tao ${ }^{1}$, Dawei Zhang ${ }^{1,2, *}$, Ruijin Hong ${ }^{1}$, \\ Yuanshen Huang ${ }^{1}$ and Daohua Zhang ${ }^{2}$ \\ 'Engineering Research Center of Optical Instrument and System, \\ Ministry of Education and Shanghai Key Lab of Modern Optical System, \\ University of Shanghai for Science and Technology, \\ No. 516 JunGong Road, Shanghai 200093, China \\ ${ }^{2}$ School of Electrical and Electronic Engineering, Nanyang Technological University, \\ 50 Nanyang Avenue, Singapore 639798, Singapore
}

(Received April 10, 2014; accepted September 18, 2014)

Key words: temperature sensor, optical fiber, refractive index, germanium coating

We report on a novel fiber-optic temperature sensor based on the temperature-

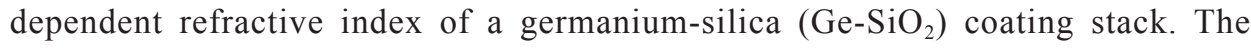
relationship between the reflectivity and the temperature-dependent refractive index of the $\mathrm{Ge}_{-} \mathrm{SiO}_{2}$ coating stack at $1550 \mathrm{~nm}$ is theoretically investigated. A reflection-type fiber-optic probe with high sensitivity and wide response range is designed and fabricated by physical vapor deposition. Experimental results show that the reflectivity is linearly dependent on temperature in the range of -30 to $130{ }^{\circ} \mathrm{C}$, and the sensor exhibits high sensitivity and structural stability in the range of 50 to $500 \mathrm{~K}$.

\section{Introduction}

Owing to its fast response, fiber-optic temperature sensors with a wide dynamic range and telemetric optical fiber transmission network have widespread applications in remote or hazardous locations, such as underground mine and tunnel construction sites. ${ }^{(1,2)}$ Owing to their invaluable advantages, various types of fiber-optic temperature sensors based on different materials and theories have been developed and fabricated. ${ }^{(3-5)}$ GaAs, $\mathrm{CdTe}$, and silicon-based sensors primarily rely on the temperature-dependent shift of the intrinsic absorption edge, whereas reflection-type optical fiber sensor systems require a grating panel. ${ }^{(6-11)}$ Generally, fiber-optic temperature sensors using a crystal plate require precise positioning and special packaging technology. ${ }^{(12-15)}$ In contrast, the process of

*Corresponding author: e-mail: dwzhang@usst.edu.cn 
coating thermo-optic films coated on the end face of an optical fiber offers a low-cost and promising technology. Semiconductor germanium $(\mathrm{Ge})$ is an ideal thermo-optic sensing material as its thermal coefficient of refractive index and thermal stability are comparable to those of the best infrared coating materials. A fiber-optic temperature sensor based on the relationship between the temperature-dependent refractive index and the absorption of Ge films has been proposed recently.(16) In this article, we report on a reflection-type fiber-optic temperature sensor based on the temperature-dependent reflectivity of a Ge$\mathrm{SiO}_{2}$ coating stack, which exhibits high stability and simplicity in sensor structure.

\section{Theoretical Model of Reflection-Type Sensor}

The key feature of the reflection-type sensor is the germanium-silica $\left(\mathrm{Ge}-\mathrm{SiO}_{2}\right)$ stack coated on the end surface of an optical fiber, in which the Ge acts as a sensor layer and the $\mathrm{SiO}_{2}$ as a protective layer, as shown in Fig. 1. On the basis of the optical admittance of the coating stacks, the intensity of the signal reflected back to the fiber core can be calculated and optimized by choosing a suitable thickness $d$ of the film stack.

The light propagation characteristics of the fiber probe with the $\mathrm{Ge}_{-} \mathrm{SiO}_{2}$ stack are studied by the equivalent conductance method. The characteristic matrices of the Ge layer and $\mathrm{SiO}_{2}$ layer for calculating the reflection of the incident light at $1550 \mathrm{~nm}$ can be described by

$$
\begin{gathered}
{\left[\begin{array}{l}
B \\
C
\end{array}\right]=\left\{\prod_{j=1}^{k}\left[\begin{array}{lr}
\cos \delta_{j} & \frac{i}{\eta_{j}} \sin \delta_{j} \\
i \eta_{j} \sin \delta_{j} & \cos \delta_{j}
\end{array}\right]\right\}\left[\begin{array}{c}
1 \\
\eta_{k+1}
\end{array}\right],} \\
R=\left(\frac{\eta_{0} B-C}{\eta_{0} B+C}\right)\left(\frac{\eta_{0} B-C}{\eta_{0} B+C}\right)^{*},
\end{gathered}
$$

where $\delta_{j}=\frac{2 \pi}{\lambda} n_{j} d_{j} \cos \theta_{j}, \eta$ is the optical admittance related to the refractive index $n$ and the incidence angle $\theta, j$ is the layer numerical order, and $k=2$.

In the optical fiber band at around $1550 \mathrm{~nm}$, the extinction coefficient can be omitted and only the change in the refractive index of Ge with temperature is considered. From

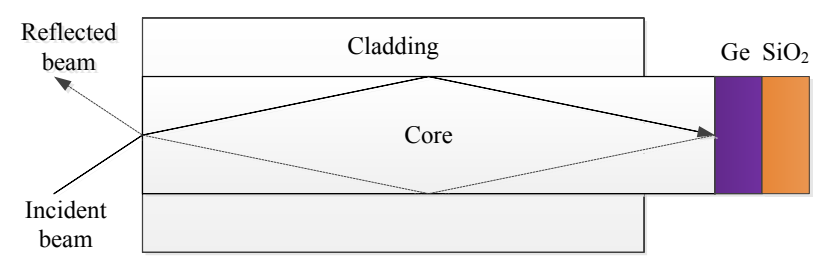

Fig. 1. (Color online) Schematic of the fiber probe of the reflection-type sensor. 
the relationship between the semiconductor band gap energy $E_{\mathrm{g}}$ and the temperature $T$, the refractive index of Ge can be expressed as ${ }^{(17)}$

$$
n=\sqrt[4]{\frac{c}{E_{\mathrm{g}}(0)-\gamma T^{2} /(T+\beta)}},
$$

where $E_{\mathrm{g}}(0)$ is the forbidden gap at $0 \mathrm{~K}$. In the range of $20-927 \mathrm{~K}, \gamma=4.774 \times 10^{-4}$ $\mathrm{eV} / \mathrm{K}, \beta=235 \mathrm{~K}, E_{\mathrm{g}}(0)=0.75 \mathrm{eV}$, and $c=224.6 \mathrm{eV}$. According to eqs. (1) and (2), the reflectivity of the stack can be expressed as a function of the thickness and thermal

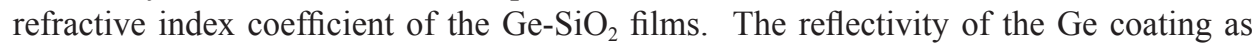
a function of film thickness at 273, 313, and $373 \mathrm{~K}$ is shown in Fig. 2. The reflectivity cyclically changes with the changes in the Ge film thickness, and the changes become more sensitive with increasing film thickness. The absorption coefficient of Ge (4.5969 $\times$ $10^{-5}$ at $1550 \mathrm{~nm}$ ) is considered to be negligible only for sufficiently small film thickness. It is found that the difference in reflectivity for a $195 \mathrm{~nm}$ thickness is approximately 0.04 from 273 to $373 \mathrm{~K}$. To minimize the absorption loss, the film thickness should be selected carefully and set in the first or second cycle.

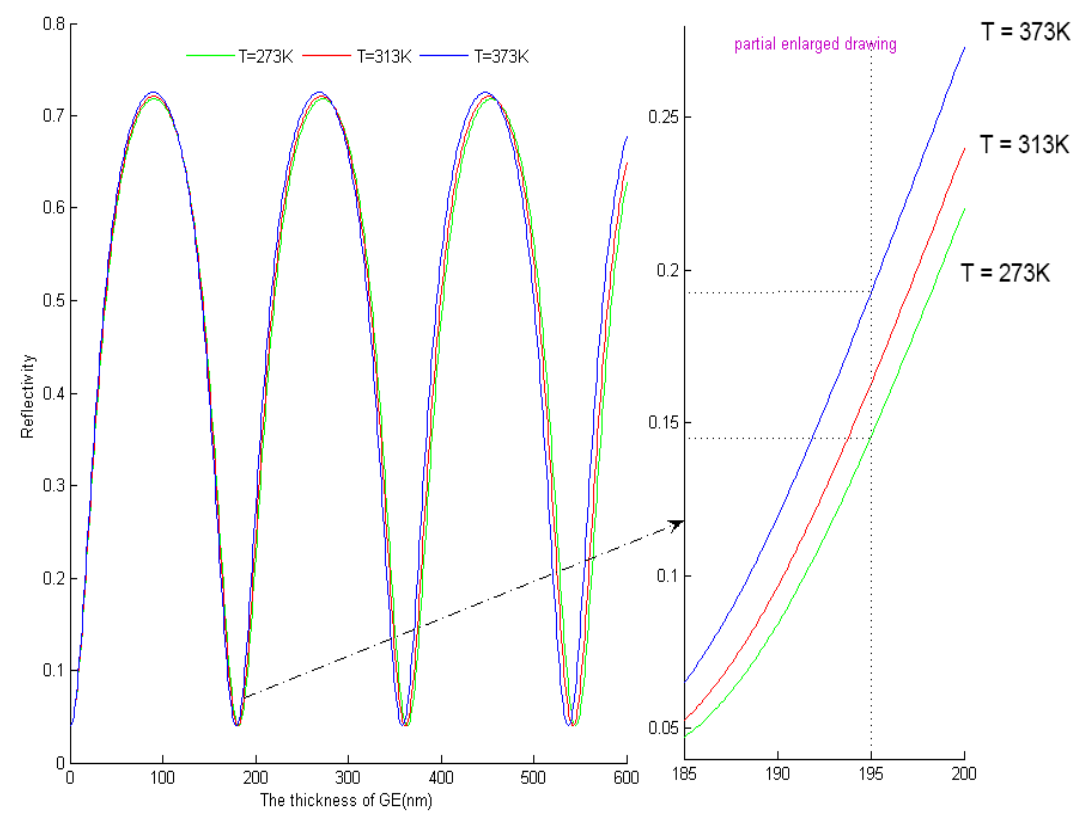

Fig. 2. (Color online) Reflectivity as a function of germanium film thickness. 


\section{Dependences of Measurement Range and Sensitivity on Thickness}

Two important characteristic parameters, namely, measurement range and sensitivity, should be evaluated for the reflection-type fiber-optic temperature sensor. The measurement range is the difference between the maximum and minimum temperatures in an approximate linear range detected by the sensor. The reflectivity of the Ge film in a fiber core medium at the interface of the fiber core and Ge is calculated on the basis of Fresnel's formula. It is found that there is periodic variation in reflectivity with

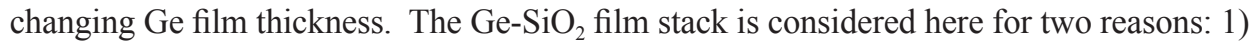
sensitivity enhancement and 2) longer operating life.

The reflectivity of the $\mathrm{Ge}_{-} \mathrm{SiO}_{2}$ film stack is dependent on the film thickness, as shown in Fig. 3. Here, the approximate cyclical change is determined by the basic interference of the film, disregarding the change in absorptivity with temperature. In fact, the definition of absorptivity via an accurate mathematical expression is difficult. However, some approximation can be achieved by carefully selecting the change in absorptivity.

Figure 4 shows the reflectivity of a fiber detector with a single Ge layer over a temperature range from 30 to $550 \mathrm{~K}$, which offers a wide measurement range. It is found that the reflectivity varies with temperature from 0.42 to 0.08 . Different from the design described above, the silica layer changed the total reflectivity at the end surface of the fiber detector, as shown in Fig. 5. While maintaining the linearity with respect to the response to temperature change, there is no degradation in the measurement range of the fiber detector with the $\mathrm{Ge}_{-} \mathrm{SiO}_{2}$ stack. In addition, the sensitivities are enhanced by decreasing the reflectivity to obtain a greater order of magnitude of the obtained signal. The final evaluation index of the fiber-optic temperature sensor in $\mathrm{dBm}$ is shown in Table 1 .

Regarding the sensitivity of the films shown in Table 1, we found that the fiber sensor with the ${\mathrm{Ge}-\mathrm{SiO}_{2}}_{2}$ film stack probe exhibits higher sensitivity than that with only a single Ge film probe.

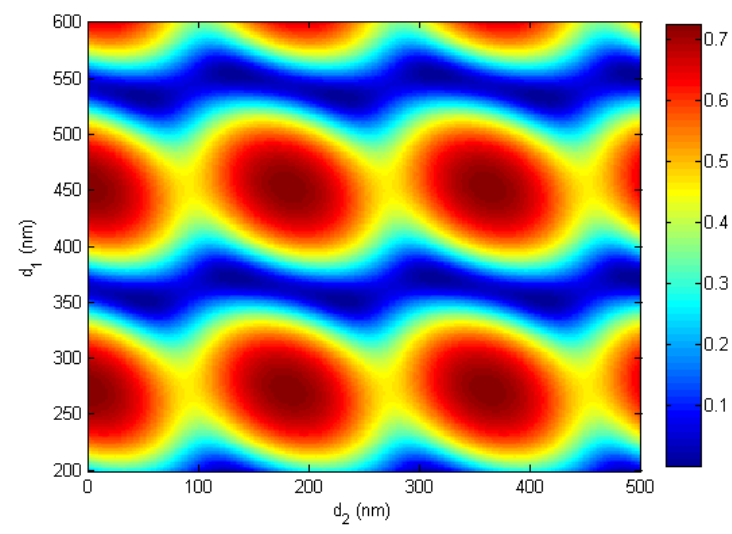

Fig. 3. (Color online) Reflectivity for various $\mathrm{Ge}\left(\mathrm{d}_{1}\right)$ and $\mathrm{SiO}_{2}\left(\mathrm{~d}_{1}\right)$ film thicknesses. 


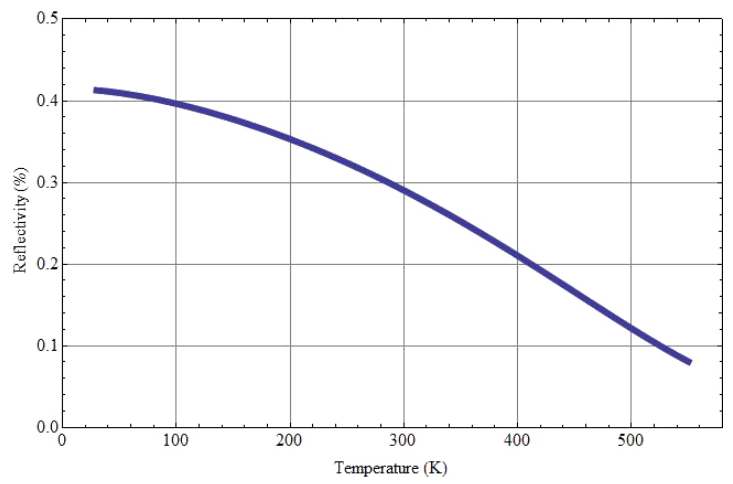

Fig. 4. (Color online) Reflectivity of a single Ge layer as a function of temperature.

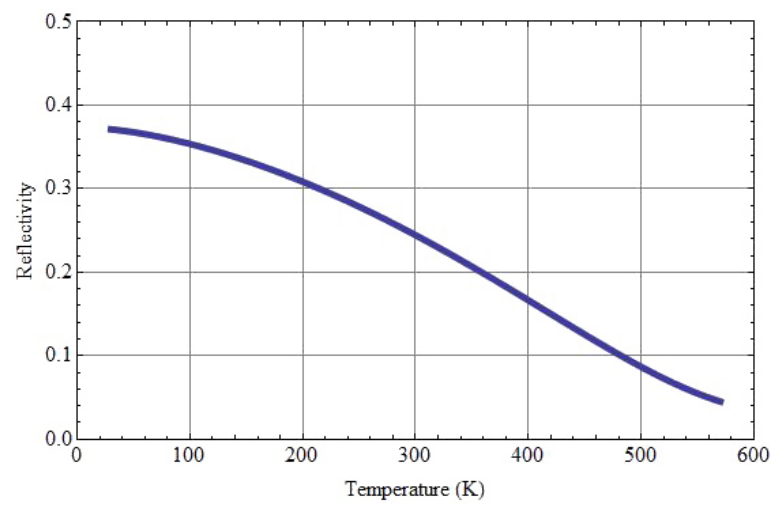

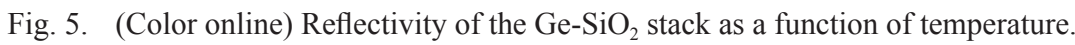

Table 1

Evaluation index of the fiber-optic temperature sensor.

\begin{tabular}{lcc}
\hline Type of sensor film & Difference in reflectivity (\%) & Difference in signal (dBm) \\
\hline Single Ge layer & 34 & 26 \\
$\mathrm{Ge} / \mathrm{SiO}_{2}$ stack & 33 & 29
\end{tabular}

This is proved by data measurement in the temperature range of 30 to $130{ }^{\circ} \mathrm{C}$, as shown in Fig. 6. Owing to cost consideration, the layer thicknesses of the $\mathrm{Ge}$ and $\mathrm{SiO}_{2}$ stack were 57.5 and $50 \mathrm{~nm}$, respectively, and deposited on the end surface of the fiber by PVD with ion beam assistance (OTFC-900, Optorun, Japan). The reflectivity power of the $\mathrm{Ge}_{-} \mathrm{SiO}_{2}$ film stack probe was approximately $4 \mathrm{~dB}$ for a temperature variation of $100{ }^{\circ} \mathrm{C}\left(-22.5 \mathrm{dBm}\right.$ at $30^{\circ} \mathrm{C} ;-26.49 \mathrm{dBm}$ at $\left.130{ }^{\circ} \mathrm{C}\right)$. Meanwhile, the reflectivity power 


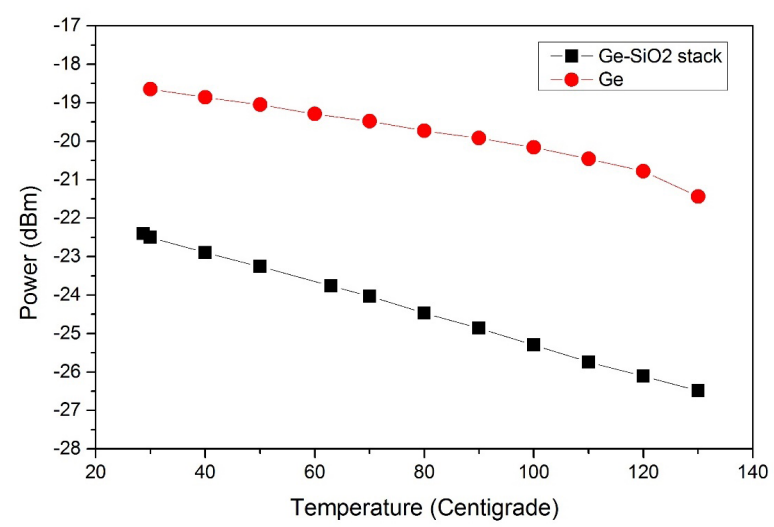

Fig. 6. (Color online) Experimental signal of fiber-optic temperature sensor.

of the fiber sensor with a single Ge film $(57.5 \mathrm{~nm})$ was $2.79 \mathrm{~dB}\left(-18.65 \mathrm{dBm}\right.$ at $30{ }^{\circ} \mathrm{C}$;

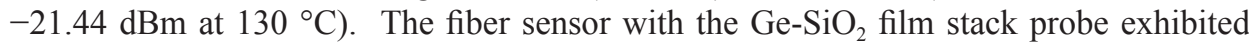
$43 \%$ higher sensitivity than that with only a single Ge film in the range of -30 to 130 ${ }^{\circ} \mathrm{C}$ and $11 \%$ in a wider range of 50 to $500 \mathrm{~K}$ theoretically. The $\mathrm{SiO}_{2}$ layer is capable of improving the sensitivity, in addition to protecting the Ge layer from oxidation and scratches.

\section{Conclusions}

We investigated theoretically and experimentally a novel fiber-optic temperature sensor based on the detection of reflection from $\mathrm{Ge}-\mathrm{SiO}_{2}$ film stacks. The $\mathrm{Ge}_{-} \mathrm{SiO}_{2}$ stack was coated directly on the end surface of the optical fiber of the fiber optic probe by physical vapor deposition. We found that the fiber sensor with a $\mathrm{Ge}_{-} \mathrm{SiO}_{2}$ film stack probe exhibited $11 \%$ higher sensitivity than that with only a single Ge film. Experimental results showed that the reflectivity exhibits a linear relationship with temperature in the range of -30 to $130{ }^{\circ} \mathrm{C}$, and the sensor is capable of operating in the 50 to $500 \mathrm{~K}$ range. This study demonstrated the development of an optical fiber with a simple structure, high stability, and sensitivity.

\section{Acknowledgements}

This work was partially supported by the National Science Instrument Important Project (2011YQ14014704, 2011YQ15004003), National High Technology Research and Development Program of China (863 Program) (2013AA030602), and Shanghai ultra-precision optical manufacturing and testing center programs from the Shanghai Committee of Science \& Technology (11DZ2290301). 


\section{References}

1 G. Yilmaz and S. E. Karlik: Sens. Actuators, A 125 (2006) 148.

2 D. A. Krohn: Fiber Optic Sensors: Fundamentals and Applications (Instrument Society of America, Research Triangle Park, North Carolina, 1988).

3 M. M. Salour, G. Schoner, M. Kull and J. H. Bechtel: Electron. Lett. 21 (1985) 135.

4 K. Kyumn, S. Tai and T. Sawada: IEEE J. Quantum Electron. 4 (1982) 676.

5 T. J. De Lyon, J. A. Roth and D. H. Chow: Vac. Sci. Technol. B 15 (1997) 329.

6 K. Kyumn, S. Tai, T. Sawada and M. Nunoshita: IEEE J. Quantum Electron. 18 (1982) 676.

7 Y. Zhao, M. Rong and Y. B. Liao: IEEE Sens. J. 3 (2003) 400.

8 M. F. Sultan and M. J. O'Rourke: Proc. SPIE 2839 (1996) 191.

9 G. Gagliardi, M. Salza, P. Ferraro, E. Chehura, R. P. Tatam, T. K. Gangopadhyay, N. Ballard, D. Paz-Soldan, J. A. Barnes, H.-P. Loock, T.-Y. Lam, J. H. Chow and P. De Natale: Sensors 10 (2010) 1823.

10 C. Francesco, P. Alfredo and S. Antonio: IEEE Sens. J. 1 (2003) 80.

11 Y. H. Hai and S. T. Uday: Laser Reflection Optical Fiber Sensor, US Patent No. 0135427 (2009).

12 C. Tanguy: J. Appl. Phys. 80 (1990) 4626.

13 J. F. Bradley, B. L. Douglas and J. M. Timothy: Proc. SPIE 6273 (2006) 62732J.

14 J. Jan, N. Bernhard and B. Pawel: J. Opt. Soc. Am. B 21 (2004) 729.

15 P. J. L. Herve and L. K. J. Vandamme: J. Appl. Phys. 77 (1995) 5476.

16 L. Min and L. L. Yu: Appl. Opt. 2 (2012) 231.

17 P. D. Li, N. G. Li and F. Yu: Nature Magazine 25 (2003) 280. 